\title{
Bounding the parameters of block-structured nonlinear feedback systems
}

\author{
V. Cerone ${ }^{1 *}$, D. Piga $^{1}$ and D. Regruto ${ }^{1}$ \\ Dipartimento di Automatica e Informatica, Politecnico di Torino, corso Duca degli Abruzzi 24, 10129 Torino, Italy
}

\section{SUMMARY}

In this paper a procedure for set-membership identification of block-structured nonlinear feedback systems is presented. Nonlinear block parameter bounds are first computed by exploiting steady-state measurements. Then, given the uncertain description of the nonlinear block, bounds on the unmeasurable inner-signal are computed. Finally, linear block parameter bounds are evaluated on the basis of output measurements and computed inner signal bounds. The computation of both the nonlinear block parameters and the inner-signal bounds is formulated in terms of semialgebraic optimization and solved by means of suitable convex LMI relaxation techniques. The problem of linear block parameter evaluation is formulated in terms of errors-invariables identification problem. Copyright (c) 0000 John Wiley \& Sons, Ltd.

Received ...

KEY WORDS: Bounded uncertainty; LMI relaxation; Block-structured nonlinear systems

\section{INTRODUCTION}

Extensive studies over the last decades in the identification of linear systems have provided a well assessed methodology for the solution of modeling problems in the time or in the frequency domain

\footnotetext{
*Correspondence to: Vito Cerone, Dipartimento di Automatica e Informatica, Politecnico di Torino, corso Duca degli Abruzzi 24, 10129 Torino, Italy. E-mail: vito.cerone@polito.it 
through either recursive or batch scheme $[1,2]$ which, unfortunately, might not be directly applied to most real-life problems that are intrinsically nonlinear. A good number of effective techniques for black-box identification of nonlinear systems can be found in the literature: polynomial NARMAX models [3], Volterra and Wiener series expansions [4], Wavelets, Neural networks and Fuzzy Logic [5] are only some examples. Although prior information on the physical structure of the system to be identified can be exploited in order to constrain the term search and to insert known nonlinearities in most of the nonlinear black-box procedures, block structured nonlinear systems provide an effective alternative to explicitly take into account a priori knowledge on the system structure (see the recent book [6] for an up-to-date collection of results and algorithms in this context). Such a class of systems can be profitably used in order to obtain simple and effective models of a wide class of nonlinear systems through suitable interconnections of memoryless nonlinear gains and linear subsystems. Nonlinearities may enter the system in different ways: either at the input or at the output end or in the feedback path around a linear model. The configuration we are dealing with in this work, also referred to as a block-structured nonlinear feedback system, is shown in Figure 1 ; it consists of a feedback system with the linear dynamic model in the forward path and the static nonlinearity in the feedback path. It must be stressed that in this context only systems which intrinsically show a nonlinear unaccessible feedback path are considered. This kind of model has been studied in the context of the well known Lur'e problem [7, 8] for which a number of results are available as far as stability is concerned. The identification of such a model solely relies on the measurement of the input and the output signal, $u_{t}$ and $y_{t}$ respectively, while all the internal signals are not assumed to be accessible.

A number of interesting applications of block-oriented feedback nonlinear models can be found in various engineering fields. This model has been successfully applied in [9] to describe the operation of dynamic mode atomic force microscopy; atomic force microscope cantilevers can be modeled as a feedback interconnection of a linear dynamic system and a nonlinear static function; the linear block describes the free cantilever dynamics while the feedback subsystem accounts for the sample interaction force, which is a nonlinear function of the tip-sample distance. In paper [10], Schoukens 


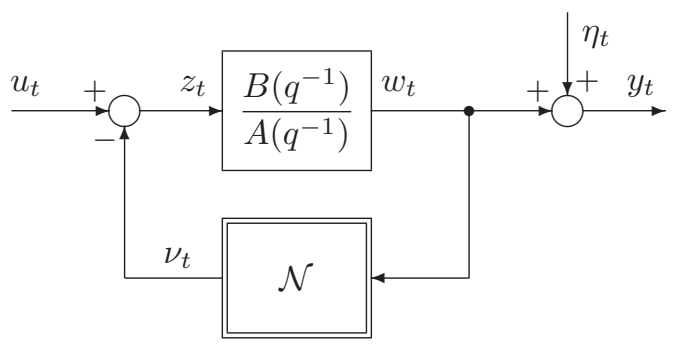

Figure 1. Block-structured nonlinear feedback system.

et al. study the identification of a block-structured nonlinear Wiener-Hammerstein system that is captured in the feedforward or the feedback path of a closed-loop system; the proposed method is applied to the identification of a microwave crystal detector. Ming-Tzu Ho and Jun-Ming Lu [11] consider the problem of synthesizing proportional-integral-derivative (PID) controllers for a given block-structured nonlinear feedback system in the presence of exogenous energy-bounded disturbance; their synthesis method is used to design a controller for a ball and wheel system apparatus. In [12], Pearson and Pottmann describes a gray-box identification approach to three classes of block-oriented models: Hammerstein models, Wiener models, and the feedback blockoriented models and show an application of the presented method to a simple first-principle model of a distillation columns. The rich diversity of hysteretic phenomena that can be generated by interconnecting a linear dynamic system with a feedback static nonlinearity, is investigated by Oh $e t$ al. in [13]. On the methodological side, further contributions to the identification of block-structured nonlinear feedback models can be found in $[14,15,16]$ while the class of block-structured systems that can be represented by a linear fractional transformation (LFT) is considered in [17, 18$]$.

A common assumption in system identification is that the measurement error is statistically described. However, there are many practical cases where reliable random variable models cannot be derived, while simple bounds are readily available. Some examples include mechanical tolerances, quantization errors in analog-to-digital converter, systematic and class errors in measurement equipments. When uncertainties are assumed to belong to a given set, a set-membership characterization of measurement errors should be preferred to the stochastic description. In this context, all parameters consistent with measurements, error bounds and the assumed model structure, are feasible solutions of the identification problem. The interested reader can find further 
details on this approach in a number of survey papers (see, e.g., $[19,20]$ ), in the book edited by Milanese et al. [21], and the special issues edited by Norton [22, 23].

In this work, we consider the identification of single-input single-output (SISO) discrete-time block-structured nonlinear feedback system when the nonlinear block can be modeled by a polynomial with finite and known order and the output measurement errors are bounded. Note that internal signals $z_{t}$ and $\nu_{t}$ are not supposed to be measurable. To the authors' best knowledge, no contribution can be found in the literature on the identification problem addressed in this paper.

The note is organized as follows. Background results on the relaxation of semialgebraic optimization problems through the theory of moments is presented in Section 2. Section 3 is devoted to the formulation of the problem. In Section 4 uncertainty intervals on the parameters of the nonlinear static block are derived, through the solution to polynomial optimization problems by exploiting steady-state input-output data. Then, in Section 5 , given the estimated uncertain nonlinearity $\mathcal{N}$ and the output measurements collected by exciting the system with an input dynamic signal, bounds on each sample of the inner signal $z_{t}$ are computed by solving two semialgebraic optimization problems. Last, in Section 6, uncertainty intervals on the parameters of the linear block are evaluated. A simulated example is reported in Section 7.

\section{NOTATION AND BACKGROUND RESULTS ON CONSTRAINED POLYNOMIAL} OPTIMIZATION

In this section we briefly review some preliminary results on the relaxation of sparse polynomial optimization problems through a hierarchy of semidefinite programming (SDP) problems. The interested reader is referred to [24] and references therein for further technical details. 


\subsection{Polynomial representation and theory of moments}

Let us denote with $\mathcal{P}_{m}^{n}[x]$ the space of real-valued polynomials of degree at most $m$ in the variable $x=\left[x_{1}, x_{2}, \ldots, x_{n}\right]^{\mathrm{T}} \in \mathbb{R}^{n}$ and let $h_{m}^{n}$ be the canonical basis of $\mathcal{P}_{m}^{n}[x]$, i.e.

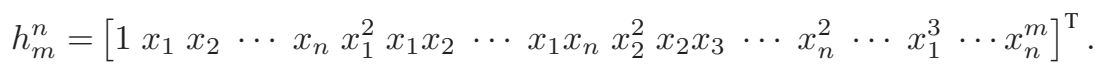

Let us define the set $\mathcal{A}_{m}^{n}=\left\{\alpha \in \mathbb{N}_{0}^{n}: \sum_{i}^{n} \alpha_{i} \leq m\right\}$, where $\alpha_{i}$ is the $i$-th component of the vector $\alpha$ and $\mathbb{N}_{0}^{n}$ denotes the set of $n$-dimensional nonnegative integers vectors. Then, the basis $h_{m}^{n}$ of the space $\mathcal{P}_{m}^{n}[x]$ can be written as $h_{m}^{n}=\left\{x^{\alpha}\right\}_{\alpha \in \mathcal{A}_{m}^{n}}$, where $x^{\alpha}=x_{1}^{\alpha_{1}} x_{2}^{\alpha_{2}} \cdots x_{n}^{\alpha_{n}}$.

Let $f$ and $g_{s}$ be in $\mathcal{P}_{m}^{n}[x]$. We denote the sequence $\mathbf{f}=\left\{f_{\alpha}\right\}_{\alpha \in \mathcal{A}_{m}^{n}}$ and $\mathbf{g}_{s}=\left\{g_{s \alpha}\right\}_{\alpha \in \mathcal{A}_{m}^{n}}$ as the coefficients of the polynomials $f$ and $g_{s}$, respectively, on the basis $h_{m}^{n}$, i.e.

$$
f(x)=\sum_{\alpha \in A_{m}^{n}} f_{\alpha} x^{\alpha}, \quad g_{s}(x)=\sum_{\alpha \in A_{m}^{n}} g_{s \alpha} x^{\alpha}
$$

Let $p=\left\{p_{\alpha}\right\}_{\alpha \in \mathcal{A}_{m}^{n}}$ be the sequence of moments (up to order $m$ ) of a probability measure $\mu$ on $\mathbb{R}^{n}$, i.e. $p_{\alpha}=\int x^{\alpha} \mu(\mathrm{d} x)$ and $M_{m}(p)$ be the truncated moment matrix associated with the distribution $\mu$, i.e. $M_{m}(p)=\int h_{m}^{n} h_{m}^{n^{\mathrm{T}}} \mu(\mathrm{d} x)$. Let us denote with $M_{m}\left(g_{k} p\right)$ the localizing matrix associated with the sequence of moments $p$ and with the polynomial $g_{k}(x)$. The interested reader is referred to [25] for details on the construction of the localizing matrix associated with a polynomial.

\section{Remark 1}

Both $M_{m}(p)$ and $M_{m}\left(g_{k} p\right)$ are square matrixes of size $\left(\begin{array}{c}n+m \\ m\end{array}\right)$.

\subsection{LMI-relaxation for polynomial optimization problems}

The LMI-relaxation technique based on the theory of moments and proposed by Lasserre in [25] is briefly reviewed here. 
Let us consider the constrained optimization problem

$$
f^{\star}=\min _{x \in \mathcal{S}} f(x),
$$

where $f \in \mathcal{P}_{m}^{n}[x]$ and $\mathcal{S} \subseteq \mathbb{R}^{n}$ is a compact semialgebraic set defined as

$$
\mathcal{S}=\left\{x \in \mathbb{R}^{n}: g_{s}(x) \geq 0, s=1, \ldots, \Xi\right\},
$$

where $g_{s}$ is a real-valued polynomial in the variable $x \in \mathbb{R}^{n}$ of degree $d_{s}=\operatorname{deg}\left(g_{s}\right)$, i.e. $g_{s} \in \mathcal{P}_{d_{s}}^{n}[x]$. Let $\delta \in \mathbb{N}$ be such that $2 \delta \geq \max \left\{m, \max _{s} d_{s}\right\}$ and $h_{2 \delta}^{n}=\left\{x^{\alpha}\right\}_{\alpha \in \mathcal{A}_{2 \delta}^{n}}$ be the canonical basis of the space $\mathcal{P}_{2 \delta}^{n}[x]$. Indeed, $f$ and $g_{s}$ belong to $\mathcal{P}_{2 \delta}^{n}[x]$.

Now, let us consider the SDP problem

$$
\begin{aligned}
& f^{\delta}=\min _{p} \sum_{\alpha \in \mathcal{A}_{2 \delta}^{n}} f_{\alpha} p_{\alpha} \\
& \text { s.t. } \quad M_{\delta}(p) \succeq 0, M_{\delta-\tilde{d}_{s}}\left(g_{s} p\right) \succeq 0, \quad s=1, \ldots, \Xi ;
\end{aligned}
$$

where $\tilde{d}_{s}=\left\lceil\frac{d_{s}}{2}\right\rceil, p=\left\{p_{\alpha}\right\}_{\alpha \in \mathcal{A}_{2 \delta}^{n}}$ is the sequence of moments up to order $2 \delta$ of some probability measure $\mu$ with support on $S$, while $M_{\delta}(p)$ is the moment matrix associated with the moments $p$ and $M_{\delta-\tilde{d}_{s}}\left(g_{s} p\right)$ is the localizing matrix associated with the polynomial $g_{s}$. Problem (4) is referred to as LMI-relaxed problem of order $\delta$ of the original polynomial problem (2). The solution $f^{\delta}$ to the convex problem (4) is a lower bound of the global optimum $f^{\star}$ of the nonconvex problem (2). Besides, under mild conditions, $f^{\delta}$ converges to $f^{\star}$ as the relaxation order $\delta$ goes to infinity. However, exact global optimum $f^{\star}$ can be obtained in practice with a reasonably low relaxation order (see [26] for a collection of test problems solved with relaxation order less or equal to 4).

Unfortunately, due to high computational complexity, the discussed LMI-relaxation is restricted to polynomial problems with a small number of optimization variables. In the next section we describe the relaxation procedure presented by Lasserre in [24] in the spirit of the work of Waki et al [27]. Such a technique exploits the sparsity in the original polynomial problems to formulate 
a sparse version of the SDP-relaxation previously described, in order to extend the applicability of such a methodology to medium and large scale problems.

\subsection{Sparse LMI-relaxation for polynomial optimization problems}

Given the optimization problem (2) with $\mathcal{S}$ as in (3), let $\mathcal{I}_{0}=\{1, \ldots, n\}$ be the union of a collection of $R$ sets $\mathcal{I}_{r} \subset\{1, \ldots, n\}$, that is $\{1, \ldots, n\}=\bigcup_{r=1}^{R} \mathcal{I}_{r}$. Further, let us partition the index set $\mathcal{S}_{0}=\{1, \ldots, \Xi\}$ into $R$ disjoint sets $S_{r}, r=1, \ldots, R$.

Let us construct the partial moment matrixes $M_{m}\left(p, \mathcal{I}_{r}\right)$ (respectively the partial localizing matrixes $\left.M_{m}\left(g_{s} p, \mathcal{I}_{r}\right)\right)$ by retaining only those rows and columns of the moment matrix $M_{m}(p)$ (respectively of the localizing matrix $M_{m}\left(g_{s} p\right)$ ), where the variables $p_{\alpha}$ are such that $\operatorname{supp}(\alpha) \in \mathcal{I}_{r}$, with $\operatorname{supp}(\alpha)$ denoting the support of the vector $\alpha$.

Now, for a given $\delta \in \mathbb{N}$ such that $2 \delta \geq \max \left\{m, \max _{s} d_{s}\right\}$, let us define the SDP problem

$$
\begin{aligned}
& f_{s p}^{\delta}=\min _{p} \sum_{\alpha \in \mathcal{A}_{2 \delta}^{n}} f_{\alpha} p_{\alpha} \\
& \text { s.t. } \quad M_{\delta}\left(p, \mathcal{I}_{r}\right) \succeq 0, \quad M_{\delta-\tilde{d}_{s}}\left(g_{s} p, \mathcal{I}_{r}\right) \succeq 0, \quad s \in \mathcal{S}_{r}, \quad r=1, \ldots, R ;
\end{aligned}
$$

The following result holds.

\section{Theorem 1}

If the indexes sets $\mathcal{I}_{r}$ and $\mathcal{S}_{r}$ are such that:

(i) for every $r=1, \ldots, R$ and for every $s \in \mathcal{S}_{r}$, the constraint $g_{s}(x) \geq 0$ defining $\mathcal{S}$ in (3), depends only on the variables $x\left(\mathcal{I}_{r}\right)=\left\{x_{i} \mid i \in \mathcal{I}_{r}\right\}$

(ii) the objective function $f$ can be written as

$$
f=\sum_{r=1}^{R} f_{r}, \text { with } f_{r} \in \mathcal{P}_{m}^{n_{r}}\left[x\left(\mathcal{I}_{r}\right)\right], \text { for every } r=1, \ldots, R,
$$

then

$$
f_{s p}^{\delta} \leq f_{s p}^{\delta+1} \leq f^{*}
$$


Besides, if there exists a value $C>0$ such that $\|x\|_{\infty} \leq C$ for all $x \in \mathcal{S}$ and the sets $\mathcal{I}_{r}$ are such that

$$
\mathcal{I}_{r+1} \cap \bigcup_{j=1}^{r} \mathcal{I}_{j} \subseteq \mathcal{I}_{q}, \text { for some } q \leq r
$$

for every $r=1, \ldots, R-1$, and hypothesis (i) an (ii) are satisfied, then

$$
\lim _{\delta \rightarrow \infty} f_{s p}^{\delta}=f^{*} .
$$

\section{Remark 2}

The size of the square matrixes $M_{m}\left(p, \mathcal{I}_{r}\right)$ and $M_{m}\left(g_{s} p, \mathcal{I}_{r}\right)$ is $\left(\begin{array}{c}n_{r}+m \\ m\end{array}\right)$, with $n_{r}$ denoting the cardinality of the set $\mathcal{I}_{r}$.

An implementation of the discussed sparse LMI-relaxation can be found in the Matlab package SparsePOP [28], which exploits the solver SeDuMi to solve semidefinite programming problems in polynomial time.

\section{PROBLEM FORMULATION}

Consider the SISO discrete-time block-structured nonlinear systems shown in Figure 1, where the linear dynamic part is modeled by a discrete-time system which transforms $z_{t}$ into the noise-free output $w_{t}$ according to

$$
w_{t}=G\left(q^{-1}\right) z_{t}=\frac{B\left(q^{-1}\right)}{A\left(q^{-1}\right)} z_{t},
$$

where $z_{t}=u_{t}-\nu_{t}$ is the unmeasurable inner signal and $A(\cdot)$ and $B(\cdot)$ are polynomials in the backward shift operator $q^{-1},\left(q^{-1} w_{t}=w_{t-1}\right)$,

$$
\begin{aligned}
& A\left(q^{-1}\right)=1+a_{1} q^{-1}+\ldots+a_{n a} q^{-n a}, \\
& B\left(q^{-1}\right)=b_{0}+b_{1} q^{-1}+\ldots+b_{n b} q^{-n b} .
\end{aligned}
$$


The nonlinear block transforms the noise-free output $w_{t}$ into $\nu_{t}$ according to

$$
\nu_{t}=\mathcal{N}\left(w_{t}, \gamma\right)=\sum_{k=1}^{n_{\gamma}} \gamma_{k} w_{t}^{k}
$$

where $n_{\gamma}$ is the polynomial degree.

In line with the work done by a number of authors, it is assumed that: (i) the steady-state gain of the linear block is not zero $[29,30]$, that is, $\sum_{j=0}^{n b} b_{j} \neq 0$; (ii) a rough upper bound of the settling time is available [31].

Let $y_{t}$ be the noise-corrupted measurements of $w_{t}$, i.e.

$$
y_{t}=w_{t}+\eta_{t}
$$

Measurements uncertainty is known to be bounded by $\Delta \eta_{t}$, i.e.,

$$
\left|\eta_{t}\right| \leq \Delta \eta_{t}
$$

Let $\gamma \in \mathbb{R}^{n_{\gamma}}$ and $\theta \in \mathbb{R}^{n_{\theta}}$ be the vectors of the unknown parameters, that is $\gamma^{\mathrm{T}}=\left[\begin{array}{lll}\gamma_{1} & \gamma_{2} & \ldots \gamma_{n_{\gamma}}\end{array}\right]$ and $\theta^{\mathrm{T}}=\left[\begin{array}{lllllll}a_{1} & \ldots & a_{n a} & b_{0} & b_{1} & \ldots & b_{n b}\end{array}\right]$, with $n_{\theta}=n a+n b+1$. The parametrization of the structure of Figure 1 is not unique. As a matter of fact, given a pair of subsystems $\tilde{G}\left(q^{-1}, \tilde{\theta}\right)$, $\tilde{\mathcal{N}}\left(w_{t}, \tilde{\gamma}\right)$, any dynamic system of the kind of the one depicted in Figure 1 where $G\left(q^{-1}, \theta\right)=$ $\tilde{G}\left(q^{-1}, \tilde{\theta}\right) /\left(1+\sigma \tilde{G}\left(q^{-1}, \tilde{\theta}\right)\right)$ and $\mathcal{N}\left(w_{t}, \gamma\right)=\tilde{\mathcal{N}}\left(w_{t}, \tilde{\gamma}\right)-\sigma w_{t}$, provides the same input-output behaviour for any constant $\sigma \in \mathbb{R}$. Such an analysis is in agreement with [15], where a structured Hammerstein-Nonlinear feedback model is considered. In order to get a unique parametrization, in this work we assume, without loss of generality, that the steady-state gain $\bar{g}$ of the linear block $G\left(q^{-1}, \theta\right)$ be 1 , that is

$$
\bar{g}=\frac{\sum_{j=0}^{n b} b_{j}}{1+\sum_{i=1}^{n a} a_{i}}=1 .
$$

In this paper we address the problem of deriving bounds on the parameters $\gamma$ and $\theta$. 


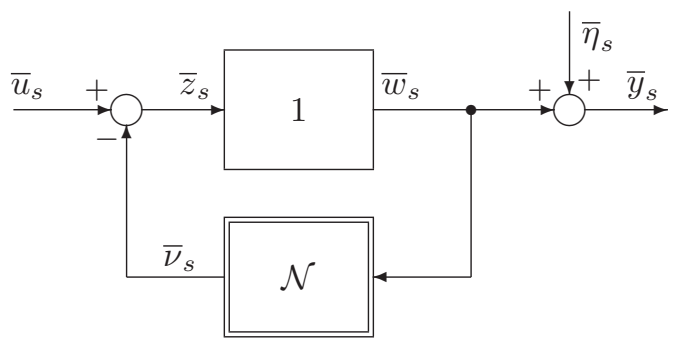

Figure 2. Steady-state behaviour of the system.

\section{BOUNDING THE NONLINEAR STATIC BLOCK PARAMETERS}

Here we exploit steady-state operating conditions to bound the parameters of the nonlinear static block. Known input and noise corrupted output sequences are collected from the steady-state response of the system to a set of step inputs with different amplitudes. We only assume to have a rough idea of the system settling time, in order to know when steady-state conditions are reached, so that steady-state data can be collected. Indeed, under condition (i) stated in Section 3, by combining equations (7), (10), (11), (12) and (13) in steady-state operating conditions we get the input-output mapping

$$
\begin{gathered}
\bar{w}_{s}=\bar{u}_{s}-\sum_{k=1}^{n} \gamma_{k} \bar{w}_{s}^{k}, s=1, \ldots, M ; \\
\bar{y}_{s}=\bar{w}_{s}+\bar{\eta}_{s}, s=1, \ldots, M
\end{gathered}
$$

where $M \geq n_{\gamma}$ is the number of measurements used in the identification of the nonlinear block, $\bar{u}^{\mathrm{T}}=\left[\bar{u}_{1}, \bar{u}_{2} \ldots \bar{u}_{M}\right]$ and $\bar{w}^{\mathrm{T}}=\left[\bar{w}_{1}, \bar{w}_{2} \ldots \bar{w}_{M}\right]$ are sequences of steady-state values of the known input signal and the unknown output signal respectively; $\bar{\eta}_{s}$ and $\bar{y}_{s}$ are the values of the noise and the measured output samples collected at some instant after the true system output has reached the steady-state value $\bar{w}_{s}$. A block diagram description of equations (14)-(15) is depicted in Figure 2. The set $\mathcal{D}_{\gamma}$ of all parameters $\gamma$ of the static nonlinear block and noise samples $\bar{\eta}$ consistent with the input-output data sequences, the assumed model structure and the error bounds is described by 
(11), (14) and (15), i.e.

$$
\begin{gathered}
\mathcal{D}_{\gamma}=\left\{(\gamma, \bar{\eta}) \in \mathbb{R}^{n_{\gamma}+M}:\left(\bar{y}_{s}-\bar{\eta}_{s}\right)+\sum_{k=1}^{n_{\gamma}} \gamma_{k}\left(\bar{y}_{s}-\bar{\eta}_{s}\right)^{k}=\bar{u}_{s},\right. \\
\left.\left|\bar{\eta}_{s}\right| \leq \Delta \bar{\eta}_{s} ; s=1, \ldots, M\right\}
\end{gathered}
$$

where $\bar{\eta}^{\mathrm{T}}=\left[\bar{\eta}_{1}, \bar{\eta}_{2}, \ldots \bar{\eta}_{M}\right]$.

For all $k=1, \ldots, n_{\gamma}$, tight bounds on each parameter $\gamma_{k}$ can be computed by solving the optimization problems

$$
\begin{aligned}
& \underline{\gamma}_{k}=\min _{\gamma, \bar{\eta} \in \mathcal{D}_{\gamma}} \gamma_{k}, \\
& \bar{\gamma}_{k}=\max _{\gamma, \bar{\eta} \in \mathcal{D}_{\gamma}} \gamma_{k} .
\end{aligned}
$$

The parameter uncertainty interval $\left(P U I_{\gamma_{k}}\right)$ on $\gamma_{k}$ is defined as

$$
P U I_{\gamma_{k}}=\left[\underline{\gamma}_{k} ; \bar{\gamma}_{k}\right]
$$

In order to guarantee well-posedness of the identification problems (17) and (18), we assume that $\mathcal{D}_{\gamma}$ is a bounded set. Then, the following condition on $\gamma$ holds:

$$
\|\gamma\|_{\infty} \leq C
$$

for some constant $C>0$ arbitrarily large.

Note that the formulated identification problems (17) and (18) are semialgebraic optimization problems since the functional is linear and the feasible set $\mathcal{D}_{\gamma}$ is defined by polynomial equalities and inequalities in the variables $\gamma_{k}$ and $\bar{\eta}_{s}$. Therefore, approximated solutions of parameter bounds can be computed through a direct implementation of the dense LMI-relaxation technique described in Section 2.2, guaranteeing monotone converge to the tight bounds $\underline{\gamma}_{k}$ and $\bar{\gamma}_{k}$. In particular, for a 
given relaxation order $\delta \geq \underline{\delta}=\left\lceil\frac{n_{\gamma}+1}{2}\right\rceil$, relaxing (17) and (18) through the dense LMI-relaxation leads to SDP problems where the number of optimization variables is $\left(\begin{array}{c}n_{\gamma}+M+2 \delta \\ 2 \delta\end{array}\right)$ and the feasible region is described by one moment matrix of size $\left(\begin{array}{c}n_{\gamma}+M+\delta \\ \delta\end{array}\right), 2 M$ localizing matrixes, each one of size $\left(\begin{array}{c}n_{\gamma}+M+\delta-\underline{\delta} \\ \delta-\underline{\delta}\end{array}\right)$ and $2 M$ localizing matrixes, each one of size $\left(\begin{array}{c}n_{\gamma}+M+\delta-1 \\ \delta-1\end{array}\right)$

Due to high computational burden and memory storage requirement, the use of the dense LMIrelaxation technique to relax (17) and (18) is limited, in practice, to identification problems with a small number $M$ of measurements, say roughly not greater than 10 . In order to handle a larger number of measurements, the peculiar structure of identification problems (17) and (18) will be analyzed to apply the sparse SDP-relaxation described in Section 2.3. To this aim, let us rewrite the feasible set $\mathcal{D}_{\gamma}$ as

$$
\begin{aligned}
& \mathcal{D}_{\gamma}=\left\{(\gamma, \bar{\eta}) \in \mathbb{R}^{n+M}:\right. \\
& g_{s}(\gamma, \bar{\eta})=\left(\bar{y}_{s}-\bar{\eta}_{s}\right)+\sum_{k=1}^{n} \gamma_{k}\left(\bar{y}_{s}-\bar{\eta}_{s}\right)^{k}-\bar{u}_{s} \geq 0, \\
& g_{s+M}(\gamma, \bar{\eta})=-\left(\bar{y}_{s}-\bar{\eta}_{s}\right)-\sum_{k=1}^{n} \gamma_{k}\left(\bar{y}_{s}+\bar{\eta}_{s}\right)^{k}+\bar{u}_{s} \geq 0, \\
& g_{s+2 M}(\gamma, \bar{\eta})=\Delta \bar{\eta}_{s}-\bar{\eta}_{s} \geq 0, \\
& g_{s+3 M}(\gamma, \bar{\eta})=\bar{\eta}_{s}+\Delta \bar{\eta}_{s} \geq 0, \\
& s=1, \ldots, M\} .
\end{aligned}
$$

The description of $\mathcal{D}_{\gamma}$ in (21) is straightforwardly obtained from (16), by rewriting each equality constraint $\left(\bar{y}_{s}-\bar{\eta}_{s}\right)+\sum_{k=1}^{n_{\gamma}} \gamma_{k}\left(\bar{y}_{s}-\bar{\eta}_{s}\right)^{k}=\bar{u}_{s}$ as two inequality constraints, i.e. 
$\left(\bar{y}_{s}-\bar{\eta}_{s}\right)+\sum_{k=1}^{n_{\gamma}} \gamma_{k}\left(\bar{y}_{s}-\bar{\eta}_{s}\right)^{k} \leq \bar{u}_{s}$ and $\left(\bar{y}_{s}-\bar{\eta}_{s}\right)+\sum_{k=1}^{n_{\gamma}} \gamma_{k}\left(\bar{y}_{s}-\bar{\eta}_{s}\right)^{k} \geq \bar{u}_{s}$.

The inherent structured sparsity of identification problems (17) and (18) is described in the following property.

\section{Property 1}

Structure of identification problems (17) and (18)

Problems (17) and (18) enjoy the features:

P 1.1

the objective function depends only on the variable $\gamma_{k}$;

$\boldsymbol{P} 1.2$

for all $s=1, \ldots, M$, the polynomials constraints $g_{s} \geq 0 g_{s+M} \geq 0$ defining $\mathcal{D}_{\gamma}$ in (21) depend only on $n_{\gamma}+1$ variables, i.e. the unknown parameters $\gamma_{k}$ and the sample noise $\bar{\eta}_{s}$;

P 1.3

for all $s=1, \ldots, M$, the constraints $g_{s+2 N} \geq 0$ and $g_{s+3 N} \geq 0$, defining $\mathcal{D}_{\gamma}$ in (21) depend only on the variable $\bar{\eta}_{s}$.

Thanks to the inherent structured sparsity of identification problems (17) and (18) described in Property 1, sparse SDP-relaxed problems for (17) and (18) can be formulated as described in the following.

Let $X \in \mathbb{R}^{n_{\gamma}+M}$ be the collection of the optimization variables for the identification problems (17) and (18), that is:

$$
X=\left[\begin{array}{l}
\gamma \\
\bar{\eta}
\end{array}\right] .
$$

Let us define the index sets $\mathcal{I}_{r}$ and $\mathcal{S}_{r}$ as

$$
\mathcal{I}_{s}=\left\{1,2, \ldots, n_{\gamma}, n_{\gamma}+s\right\} \text { for } s=1, \ldots, M
$$




$$
\mathcal{S}_{s}=\{s, s+M, s+2 M, s+3 M\} \text { for } s=1, \ldots, M \text {. }
$$

The index sets $\mathcal{I}_{s}$ and $\mathcal{S}_{s}$ are constructed on the basis of the sparse structure of the identification problems (17) and (18) highlighted by Property 1 . More precisely, the sets $\mathcal{I}_{s}$ and $\mathcal{S}_{s}$ are such that, for all $r \in \mathcal{S}_{s}$, all the polynomial constraints $g_{r} \geq 0$ in the definition of $\mathcal{D}_{\gamma}$ depend only on the variables $X_{i}$, with $i \in \mathcal{I}_{s}$.

Now, for a given relaxation order $\delta \geq \underline{\delta}$, let us consider the SDP problems:

$$
\begin{aligned}
& \underline{\gamma}_{k}^{\delta}=\min _{p \in \mathcal{D}_{\gamma}^{\delta}} \sum_{\alpha \in \mathcal{A}_{2 \delta}^{n_{\gamma}+M}} \Gamma_{k \alpha} p_{\alpha}, \\
& \bar{\gamma}_{k}^{\delta}=\max _{p \in \mathcal{D}_{\gamma}^{\delta}} \sum_{\alpha \in \mathcal{A}_{2 \delta}^{n_{\gamma}+M}} \Gamma_{k \alpha} p_{\alpha},
\end{aligned}
$$

where $\Gamma_{k}=\left\{\Gamma_{k \alpha}\right\}_{\alpha \in \mathcal{A}_{2 \delta}^{n_{\gamma}+M}}$ is the vector of the coefficients of $\gamma_{k}$ in the basis $h_{2 \delta}^{n_{\gamma}+M}=$ $\left\{X^{\alpha}\right\}_{\alpha \in A_{2 \delta}^{n_{\gamma}+M}}$, which is the canonical basis of the real-valued polynomials of degree $2 \delta$ in the variables vector $X$ defined in (22). The feasible region $\mathcal{D}_{\gamma}^{\delta}$ for problems (25) and (26) is a convex set defined as

$$
\begin{aligned}
\mathcal{D}_{\gamma}^{\delta}=\{p: & M_{\delta}\left(p, \mathcal{I}_{s}\right) \succeq 0, \\
& \left.M_{\delta-\tilde{d}_{r}}\left(g_{r} p, \mathcal{I}_{s}\right) \succeq 0, r \in \mathcal{S}_{s}, s=1, \ldots, M\right\}
\end{aligned}
$$

where $M_{\delta}\left(p, \mathcal{I}_{s}\right)$ is the moment matrix of order $\delta$ associated with the variables $X\left(\mathcal{I}_{s}\right)$; $M_{\delta-\tilde{d}_{r}}\left(g_{r} p, \mathcal{I}_{s}\right)$ is the localizing matrix associated with the variables $X\left(\mathcal{I}_{s}\right)$ and the constraint $g_{r} \geq 0$ defining $\mathcal{D}_{\gamma}$ in (21), and $\tilde{d}_{r}=\left\lceil\frac{d_{r}}{2}\right\rceil$, with $d_{r}$ denoting the degree of the polynomial $g_{r}$. Indeed, $\tilde{d}_{r}=\left\lceil\frac{n_{\gamma}+1}{2}\right\rceil$ for $r=1, \ldots, 2 M$, while $\tilde{d}_{r}=1$ for $r=2 M+1, \ldots, 4 M$.

Let us define the $\delta$-relaxed uncertainty intervals as

$$
P U I_{\gamma_{k}}^{\delta}=\left[\underline{\gamma}_{k}^{\delta} ; \bar{\gamma}_{k}^{\delta}\right]
$$




\section{Property 2}

For every $k=1, \ldots, n_{\gamma}$, the $\delta$-relaxed parameter uncertainty interval $P U I_{\gamma_{k}}^{\delta}$ satisfies the following properties.

\section{P 2.1}

Guaranteed relaxed uncertainty intervals. For any relaxation order $\delta \geq \underline{\delta}$, the $\delta$-relaxed parameter uncertainty interval $P U I_{\gamma_{k}}^{\delta}$ is guaranteed to contain the true parameter $\gamma_{k}$ to be estimated, i.e.

$$
\gamma_{k} \in P U I_{\gamma_{k}}^{\delta}
$$

\section{$\boldsymbol{P} 2.2$}

Monotone convergence to tight uncertainty intervals. For any relaxation order $\delta \geq \underline{\delta}$, the $\delta$ relaxed parameter uncertainty interval $P U I_{\gamma_{k}}^{\delta}$ becomes tighter as the relaxation order $\delta$ increases, that is

$$
P U I_{\gamma_{k}}^{\delta+1} \subseteq P U I_{\gamma_{k}}^{\delta}
$$

Besides, the interval $P U I_{\gamma_{k}}^{\delta}$ converges to the tight interval $P U I_{\gamma_{k}}$ as the LMI relaxation order goes to infinity, that is:

$$
\begin{aligned}
& \lim _{\delta \rightarrow \infty} \underline{\gamma}_{k}^{\delta}=\underline{\gamma}_{k} \\
& \lim _{\delta \rightarrow \infty} \bar{\gamma}_{k}^{\delta}=\bar{\gamma}_{k} .
\end{aligned}
$$

Proof Index sets $\mathcal{I}_{s}$ and $\mathcal{S}_{s}$ defined in (23) and (24) were carefully constructed in such a way that the assumptions of Theorem 1 are satisfied. Furthermore, from conditions (12) and (20), the infinity norm $\|X\|_{\infty}$ of the variables vector $X$ is always bounded. Therefore, by applying the first part of Theorem 1 to the identification problems (17)-(18) and to the corresponding SDP-relaxed problems 
(25)-(26) we get:

$$
\begin{aligned}
& \underline{\gamma}_{k}^{\delta} \leq \underline{\gamma}_{k}^{\delta+1} \leq \underline{\gamma}_{k} \\
& \bar{\gamma}_{k}^{\delta} \geq \bar{\gamma}_{k}^{\delta+1} \geq \bar{\gamma}_{k}
\end{aligned}
$$

Then, from the definition of the intervals $P U I_{\gamma_{k}}$ and $P U I_{\gamma_{k}}^{\delta}$ and equations (33)-(34), we get

$$
\gamma_{k} \in P U I_{\gamma_{k}} \subseteq P U I_{\gamma_{k}}^{\delta+1} \subseteq P U I_{\gamma_{k}}^{\delta}
$$

as stated in P. 2.1 and in the first part of P. 2.2. Besides, from the second part of Theorem 1, convergence conditions given in the second part of Property P. 2.2 follow.

As to the computational complexity, the number of decision variables for the SDP problems

(25) and (26) is $M\left(\begin{array}{c}n_{\gamma}+1+2 \delta \\ 2 \delta\end{array}\right)-(M-1)\left(\begin{array}{c}n_{\gamma}+2 \delta \\ 2 \delta\end{array}\right)$, while the feasible region $\mathcal{D}_{\gamma}^{\delta}$ is described by: $M$ moment matrixes, each one of size $\left(\begin{array}{c}n_{\gamma}+1+\delta \\ \delta\end{array}\right) ; 2 M$ localizing matrixes, each one of size $\left(\begin{array}{c}n_{\gamma}+1+\delta-\underline{\delta} \\ \delta-\underline{\delta}\end{array}\right)$, associated with the $\left(n_{\gamma}+1\right)$-degree polynomial constraints $g_{s} \geq 0$ and $g_{s+M} \geq 0$ defining $\mathcal{D}_{\gamma} ; 2 M$ localizing matrixes, each one of size $\left(\begin{array}{c}n_{\gamma}+\delta \\ \delta-1\end{array}\right)$, associated with linear constraints $g_{s+2 M} \geq 0$ and $g_{s+3 M} \geq 0$ defining $\mathcal{D}_{\gamma}$.

\section{BOUNDING THE UNMEASURABLE INNER SIGNAL $z_{t}$}

In the second stage of our procedure we stimulate the block-structured nonlinear feedback system of Figure 1 with a persistently exciting input signal $u_{t}$ and evaluate bounds on each sample of the corresponding unmeasurable inner signal $z_{t}$. For each input sample $u_{t}$, bounds on the corresponding Copyright (c) 0000 John Wiley \& Sons, Ltd.

Int. J. Robust. Nonlinear Control (0000) 
inner unmeasurable signal sample $z_{t}$ can be evaluated through

$$
\underline{z}_{t}=u_{t}-\bar{\nu}_{t}, \quad \bar{z}_{t}=u_{t}-\underline{\nu}_{t}
$$

where

$$
\underline{\nu}_{t}=\min _{\mathcal{D}_{\gamma \eta}^{t}} \sum_{k}^{n_{\gamma}} \gamma_{k}\left(y_{t}-\eta_{t}\right)^{k},
$$

and

$$
\bar{\nu}_{t}=\max _{\mathcal{D}_{\gamma \eta}^{t}} \sum_{k}^{n_{\gamma}} \gamma_{k}\left(y_{t}-\eta_{t}\right)^{k} .
$$

The feasible region $\mathcal{D}_{\gamma \eta}^{t}$ for problems (37) and (38) is the cartesian product of the set $\mathcal{D}_{\gamma}$ and the set of noise samples $\eta_{t}$ satisfying the a priori hypothesis (12) on the error bounds, i.e.

$$
\begin{gathered}
\mathcal{D}_{\gamma \eta}^{t}=\left\{\left(\gamma, \bar{\eta}, \eta_{t}\right) \in \mathbb{R}^{n_{\gamma}+M+1}:\left(\bar{y}_{s}-\bar{\eta}_{s}\right)+\sum_{k=1}^{n_{\gamma}} \gamma_{k}\left(\bar{y}_{s}-\bar{\eta}_{s}\right)^{k}=\bar{u}_{s},\right. \\
\left.\left|\eta_{t}\right| \leq \Delta \eta_{t} ; \quad\left|\bar{\eta}_{s}\right| \leq \Delta \bar{\eta}_{s} ; s=1, \ldots, M\right\}
\end{gathered}
$$

Since the objective function for (37) and (38) is polynomial and the feasible region $\mathcal{D}_{\gamma \eta}^{t}$ is defined by polynomial constraints, (37) and (38) are semialgebraic optimization problems. Besides, like (17) and (18), problems (37) and (38) show an inherent structured sparsity. In fact, the objective function depends only on $n_{\gamma}+1$ variables, i.e. the unknown nonlinear block parameters $\gamma$ and the output noise $\eta_{t}$, while the linear constraints $\left|\bar{\eta}_{s}\right| \leq \Delta \bar{\eta}_{s}$ and $\left|\eta_{t}\right| \leq \Delta \eta_{t}$ defining the feasible region $\mathcal{D}_{\gamma \eta}^{t}$ depend only on the sample disturbances $\bar{\eta}_{s}$ and $\eta_{t}$, respectively. Therefore, guaranteed bounds on the signal $\nu_{t}$ (and consequently on the inner signal $z_{t}$ ) can be computed through the same LMIrelaxation method used in Section 4 for bounding the nonlinear block parameters. Similar results presented in Property 2 hold as far as the computation of bounds on $\nu_{t}$ and $z_{t}$ is concerned. 


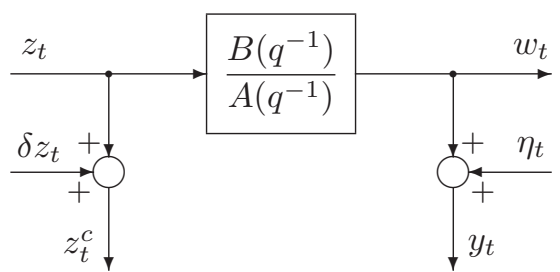

Figure 3. Errors-in-variables setup for bounding the parameters of the linear system.

\section{BOUNDING THE PARAMETERS OF THE LINEAR DYNAMIC MODEL}

Given bounds $\underline{z}_{t}$ and $\bar{z}_{t}$ computed in the second stage of the procedure, a compact description of the inner unmeasurable signal $z_{t}$ in terms of its central value $z_{t}^{c}$ and its perturbation $\delta z_{t}$ is given by

$$
z_{t}=z_{t}^{c}-\delta z_{t}
$$

where

$$
z_{t}^{c}=\frac{\bar{z}_{t}+\underline{z}_{t}}{2}
$$

Indeed, the perturbation $\delta z_{t}$ is such that

$$
\left|\delta z_{t}\right| \leq \Delta z_{t}
$$

with

$$
\Delta z_{t}=\frac{\bar{z}_{t}-\underline{z}_{t}}{2}
$$

Thanks to such a description of the unknown signal $z_{t}$, we can formulate the identification of the linear model in terms of the noisy output sequence $\left\{y_{t}\right\}$ and the uncertain inner sequence $\left\{z_{t}\right\}$ as shown in Figure 3.

Such a formulation is commonly referred to as a bounded errors-in-variables (EIV) problem, i.e. a parameter estimation in a linear-in-parameter model where both input and output measurements are corrupted by bounded noise.. As a matter of fact, by combining equations (7)-(11) and (40) we 
get

$$
y_{t}=-\sum_{i=1}^{n a}\left(y_{t-i}-\eta_{t-i}\right) a_{i}+\sum_{j=0}^{n b}\left(z_{t-j}^{c}-\delta z_{t-j}\right) b_{j}+\eta_{t} .
$$

The feasible parameters set $\mathcal{D}_{\theta}$ for the linear system is then defined by equation (44) and by conditions (12), (13) and (42), i.e.

$$
\begin{aligned}
\mathcal{D}_{\theta} & =\left\{(\theta, \eta) \in \mathbb{R}^{n_{\theta}+N}: y_{t}=-\sum_{i=1}^{n a}\left(y_{t-i}-\eta_{t-i}\right) a_{i}+\sum_{j=0}^{n b}\left(z_{t-j}^{c}-\delta z_{t-j}\right) b_{j}+\eta_{t} ;\right. \\
\bar{g} & \left.=1 ;\left|\eta_{t}\right| \leq \Delta \eta_{t} ;\left|\delta z_{t}\right| \leq \Delta z_{t} ; t=1, \ldots, N\right\},
\end{aligned}
$$

where $N$ is the length of the persistently exciting input sequence $\left\{u_{t}\right\}$ used in the second stage of the identification procedure. Then, bounds on the linear block parameters $\theta_{j}$ can be computed for all $j=1, \ldots, n_{\theta}$ by solving the nonconvex optimization problems

$$
\begin{aligned}
& \underline{\theta}_{j}=\min _{\theta, \eta \in \mathcal{D}_{\theta}} \theta_{j}, \\
& \bar{\theta}_{j}=\max _{\theta, \eta \in \mathcal{D}_{\theta}} \theta_{j} .
\end{aligned}
$$

Guaranteed uncertainty intervals $P U I_{\theta_{j}}=\left[\underline{\theta}_{j} ; \bar{\theta}_{j}\right]$ on the parameters $\theta_{j}$ can be numerically computed by exploiting the methods described in [32] and in the recent works [33, 34], where efficient convex-relaxation procedures are proposed to compute bounds on the parameters of linear systems in the EIV framework when measured data are affected by bounded noise. It must be pointed out that, if the linear block $G\left(q^{-1}\right)$ is known to be stable, stability constraints on the linear system parameters $a_{1}, \ldots, a_{n a}$ can be imposed in the definition of the set $\mathcal{D}_{\theta}$, as described in [35], in order to improve the accuracy in evaluating the uncertainty intervals $P U I_{\theta_{j}}$.

\section{A SIMULATED EXAMPLE}

In this section we illustrate the discussed parameter bounding procedure through a numerical example. The system considered here is characterized by (8), (9) and (10), with $\gamma^{\mathrm{T}}=\left[\begin{array}{lll}\gamma_{1} & \gamma_{2} & \gamma_{3}\end{array}\right]=$ 
$\left[\begin{array}{lll}-1.1 & 0.8 & 1.1\end{array}\right]$ and $\theta^{\mathrm{T}}=\left[\begin{array}{llll}a_{1} & a_{2} & b_{1} & b_{2}\end{array}\right]=\left[\begin{array}{llll}-1.3114 & 0.3679 & 0.1713 & -0.1148\end{array}\right]$. Two different simulations are performed. In the first one, a small data set is used during the identification. In particular, the length of steady-state and the transient data sequence is $M=30$ and $N=80$, respectively. In the second simulation, $M=300$ and $N=1000$ are chosen. The steady-state input sequence $\left\{\bar{u}_{s}\right\}$ belongs to the interval $[-10,+10]$, while the transient input sequence $\left\{u_{t}\right\}$ belongs to the uniform distribution $U[-10,+10]$. The output noises $\bar{\eta}_{s}$ and $\eta_{t}$ are random variables belonging to the uniform distributions $U\left[-\Delta \eta_{t},+\Delta \eta_{t}\right]$ and $U\left[-\Delta \bar{\eta}_{s},+\Delta \bar{\eta}_{s}\right]$, respectively. Bounds on steadystate and transient output measurement errors have the same value, i.e., $\Delta \eta_{t}=\Delta \bar{\eta}_{s} \triangleq \Delta \eta$. The chosen value of $\Delta \eta$ is such that the signal to noise ratio $\overline{S N R}$ in the steady-state sequence, defined as

$$
\overline{S N R}=10 \log \frac{\sum_{s=1}^{M} \bar{w}_{s}^{2}}{\sum_{s=1}^{M} \bar{\eta}_{s}^{2}},
$$

is $29 \mathrm{db}$ in both simulations, while the signal to noise ratio $S N R$ in the transient sequence, defined as

$$
S N R=10 \log \frac{\sum_{t=1}^{N} w_{t}^{2}}{\sum_{t=1}^{N} \eta_{t}^{2}}
$$

is $27 \mathrm{db}$ when $N=80$ and $28 \mathrm{db}$ when $N=1000$.

Bounds on the nonlinear block parameters $\gamma$ are evaluated by solving (25) and (26) for a relaxation order $\delta=3$. It is worth remarking that in the considered example, and for steady-state data sequence of length $M=30$, the number of decision variables for the optimization problems (25)-(26) is 3,864 and the feasible region is defined by 60 moment matrixes of size 35 ; 60 localizing matrixes of size 5 and 30 localizing matrixes of size 15 . On the other hand, if the sparsity was not taken into account in the relaxation of (17) and (18), the number of optimization variable for the corresponding SDPrelaxed problems would be more than 3 million, while the feasible region would be described by a moment matrix of size 7,140; 60 localizing matrixes of size 34 and 60 localizing matrixes of size 595 , leading to an untractable optimization problem.

By exploiting the same LMI-relaxation approach used in the computation of the uncertainty intervals on $\gamma$, bounds on the inner signals $z_{t}$ (for $t=1, \ldots, N$ ) are evaluated by relaxing problems (37) 
and (38) for an LMI-relaxation order $\delta=3$. Finally, uncertainty intervals on the linear block parameters $\theta$ are evaluated by solving the formulated error-in-variables problems (46) and (47) with the algorithm proposed in [33] and by enforcing stability constraints on the linear system $G\left(q^{-1}\right)$ as in [35]. Results on the evaluation of nonlinear and the linear block parameters are reported, respectively, in Table I and II, which show the obtained parameter bounds, the parameter uncertainties $\Delta \gamma_{k}^{\delta}$ and $\Delta \theta_{j}$, together with the central estimates $\gamma_{k}^{c, \delta}$ and $\theta_{j}^{c}$, defined as

$$
\begin{gathered}
\Delta \gamma_{k}^{\delta}=\frac{\bar{\gamma}_{k}^{\delta}-\underline{\gamma}_{k}^{\delta}}{2}, \quad \Delta \theta_{j}=\frac{\bar{\theta}_{j}-\underline{\theta}_{j}}{2}, \\
\gamma_{k}^{c, \delta}=\frac{\bar{\gamma}_{k}^{\delta}+\underline{\gamma}_{k}^{\delta}}{2}, \quad \theta_{j}^{c}=\frac{\bar{\theta}_{j}+\underline{\theta}_{j}}{2} .
\end{gathered}
$$

Results in Tables I and II show that, as expected, the true parameters values are included in the computed uncertainty intervals. Furthermore, the presented procedure provides satisfactory uncertainty intervals on both the linear and the nonlinear block parameters also when a small data set is used for the identification.

Table I. Nonlinear block. - Parameter central estimates $\left(\gamma_{k}^{c, \delta}\right)$, parameter bounds $\left(\underline{\gamma}_{k}^{\delta}, \bar{\gamma}_{k}^{\delta}\right)$ and parameter uncertainty bounds $\Delta \gamma_{k}^{\delta}$ for relaxation order $\delta=3$ for steady-state data sequence of length $M=30$ and $M=300$.

\begin{tabular}{ccccccc}
\hline \multirow{2}{*}{$M$} & Parameter & $\begin{array}{c}\text { True } \\
\text { value }\end{array}$ & $\underline{\gamma}_{k}^{\delta}$ & $\gamma_{k}^{c, \delta}$ & $\bar{\gamma}_{k}^{\delta}$ & $\Delta \gamma_{k}^{\delta}$ \\
\hline \multirow{2}{*}{30} & $\gamma_{1}$ & -1.1000 & -1.1270 & -0.9989 & -0.8708 & 0.1281 \\
& $\gamma_{2}$ & 0.8000 & 0.7866 & 0.8236 & 0.8606 & 0.0370 \\
& $\gamma_{3}$ & 1.1000 & 0.9958 & 1.0642 & 1.1327 & 0.0684 \\
\hline \multirow{2}{*}{300} & & & & & & \\
& $\gamma_{1}$ & -1.1000 & -1.1072 & -1.0984 & -1.0895 & 0.0088 \\
& $\gamma_{2}$ & 0.8000 & 0.7959 & 0.8012 & 0.8065 & 0.0053 \\
& $\gamma_{3}$ & 1.1000 & 1.0957 & 1.1003 & 1.1050 & 0.0046 \\
\hline
\end{tabular}


Table II. Linear block. - Parameter central estimates $\left(\theta_{j}^{c}\right)$, parameter bounds $\left(\underline{\theta}_{j}, \bar{\theta}_{j}\right)$ and parameter uncertainty bounds $\Delta \theta_{j}$ for transient data sequence of length $N=80$ and $N=1000$.

\begin{tabular}{|c|c|c|c|c|c|c|}
\hline$N$ & Parameter & $\begin{array}{c}\text { True } \\
\text { value }\end{array}$ & $\underline{\theta}_{j}$ & $\theta_{j}^{c}$ & $\bar{\theta}_{j}$ & $\Delta \theta_{j}$ \\
\hline \multirow[t]{4}{*}{80} & $\theta_{1}$ & -1.3114 & -1.6986 & -1.3414 & -0.9842 & 0.3572 \\
\hline & $\theta_{2}$ & 0.3679 & 0.1014 & 0.4000 & 0.6986 & 0.2986 \\
\hline & $\theta_{3}$ & 0.1713 & 0.1635 & 0.1747 & 0.1860 & 0.0113 \\
\hline & $\theta_{4}$ & -0.1148 & -0.1761 & -0.1171 & -0.0580 & 0.0591 \\
\hline \multirow[t]{4}{*}{1000} & $\theta_{1}$ & -1.3114 & -1.5340 & -1.3215 & -1.1091 & 0.2125 \\
\hline & $\theta_{2}$ & 0.3679 & 0.1979 & 0.3793 & 0.5606 & 0.1814 \\
\hline & $\theta_{3}$ & 0.1713 & 0.1656 & 0.1718 & 0.1779 & 0.0062 \\
\hline & $\theta_{4}$ & -0.1148 & -0.1453 & -0.1133 & -0.0813 & 0.0320 \\
\hline
\end{tabular}

\section{CONCLUSIONS}

A computationally tractable procedure is presented for parameter bounds computation of blockstructured nonlinear feedback systems. First, the computation of nonlinear block parameter bounds is formulated in terms of sparse polynomial optimization problems, whose approximated solutions are computed by means of LMI-relaxation techniques. The peculiar structure of the formulated optimization problems is exploited to reduce the computational complexity of the corresponding LMI-relaxed problems. The parameter uncertainty intervals computed by solving the relaxed problems are proven to contain the unknown parameters to be estimated. Besides, such parameter bounds are proven to monotonically converge to the tight ones as the relaxation order goes to infinity. Analogous results also hold for the computation of bounds on the unmeasurable inner signal. By using the inner signal bounds, the problem of bounding the linear block parameters is formulated in terms of errors-in-variables identification with bounded errors, and it is solved through the techniques available in the literature. The numerical example shows that the proposed procedure can be used in medium and large scale identification problems. Anyway, satisfactory uncertainty intervals on both the linear and nonlinear block parameters are obtained also for a small data set.

\section{REFERENCES}


1. Ljung L. System Identification, Theory for the User. Prentince Hall: Upper Saddle River, 1999.

2. Söderström T, Stoica P. System Identification. Prentice Hall: Upper Saddle River, 1989.

3. Leonaritis IJ, Billings SA. Input-output parametric models for nonlinear systems. International Journal of Control $1985 ; 41: 303-344$.

4. Rugh W. Nonlinear System Theory: The Volterra/Wiener Approach. Johns Hopkins Univ. Press: Baltimore, 1981.

5. Sjöberg J, Zhang Q, Ljung L, Benveniste A, Delyon B, Glorennec P, Hjalmarsson H, Juditsky A. Nonlinear blackbox modeling in system identification: a unified overview. Automatica 1995; 31(12):1691-1724.

6. Bai E, Giri F. Block-oriented nonlinear system identification. Lecture notes in Control and Information sciences, Springer: Berlin, 2010.

7. Vidyasagar M. Nonlinear Systems Analysis. Prentice Hall: Englewood Cliffs, NJ, 1978.

8. Khalil HK. Nonlinear Systems. Prentice Hall: Upper Saddle River, NJ, 2000.

9. Basso M, Materassi D, Salapaka M. Hysteresis models of dynamic mode atomic force microscopes: analysis and identification via harmonic balance. Nonlinear Dynamics 2008; 54:297-306.

10. Schoukens J, Gommé L, Moer WV, Rolain Y. Identification of a block-structured nonlinear feedback system, applied to a microwave crystal detector. IEEE Trans. Instrumentation and Measurement 2008; 57(8):1734-1740.

11. Ho MT, Lu JM. $H_{\infty}$ PID controller design for Lur'e systems and its application to a ball and wheel apparatus. Int. J. of Control 2005; 78(1):53-64.

12. Pearson RK, Pottmann M. Gray-box identification of block-oriented nonlinear models. J. of Process Control 2000; 10:301-315.

13. Oh J, Drincic B, Bernstein DS. Nonlinear feedback models of hysteresis. IEEE Control Systems Magazine 2009; 29(1):100-119.

14. Billings SA, Fakhouri SY. Identification of systems containing linear dynamic and static nonlinear elements. Automatica 1982; 18(1):15-26.

15. Pelt THV, Bernstein DS. Nonlinear system identification using Hammerstein and nonlinear feedback models with piecewise linear static map. Int. J. of Control 2001; 74(18):1807-1823.

16. Lauwers L, Schoukens J, Pintelon R, Enqvist M. A nonlinear block structure identification procedure using frequency response function measurement. IEEE Trans. Instrumentation and Measurement 2008; 57(10):22572264.

17. Hsu K, Poolla K, Vincent T. Identification of structured nonlinear systems. IEEE Transaction on Automatic Control 2008; 53(11):2497-2513.

18. Pepona E, Paoletti S, Garulli A, Date P. Identification of piecewise affine LFR models of interconnected systems. IEEE Transaction on Control System Technology 2011; 19(1):148-155.

19. Milanese M, Vicino A. Optimal estimation theory for dynamic sistems with set membership uncertainty: an overview. Automatica 1991; 27(6):997-1009. 
20. Walter E, Piet-Lahanier H. Estimation of parameter bounds from bounded-error data: a survey. Mathematics and Computers in simulation 1990; 32:449-468.

21. Milanese M, Norton J, Piet-Lahanier H, Walter E ( (eds.)). Bounding approaches to system identification. Plenum Press: New York, 1996.

22. Norton (Ed) J. Special issue on bounded-error estimation. Int. J. of Adapt. Control \& Sign. Proces. 1994; 8(1).

23. Norton (Ed) J. Special issue on bounded-error estimation. Int. J. of Adapt. Control \& Sign. Proces. 1995; 9(1).

24. Lasserre JB. Convergent semidefinite relaxations in polynomial optimization with sparsity. SIAM Journal on Optimization 2006; 17(1):822-843.

25. Lasserre JB. Global optimization with polynomials and the problem of moments. SIAM Journal on Optimization 2001; 11:796-817.

26. Henrion D, Lasserre JB. Solving nonconvex optimization problems. IEEE Control Systems Magazine 2004; 24(3):72-83.

27. Waki H, Kim S, Kojima M, Muramatsu M. Sums of squares and semidefinite programming relaxations for polynomial optimization problems with structured sparsity. SIAM Journal on Optimization 2006; 17(1):218-242.

28. Waki H, Kim S, Kojima M, Muramatsu M, Sugimoto H. SparsePOP: a sparse semidefinite programming relaxation of polynomial optimization problems. ACM Transaction on Mathematical Software 2008; 35(2):1-13.

29. Lang Z. Controller design oriented model identification method for Hammerstein system. Automatica 1993; 29(3):767-771.

30. Sun L, Liu W, Sano A. Identification of a dynamical system with input nonlinearity. IEE Proc. Part D 1999; 146(1):41-51.

31. Kalafatis A, Wang L, Cluett W. Identification of Wiener-type nonlinear systems in a noisy enviroment. Int. J. Control 1997; 66(6):923-941.

32. Cerone V. Parameter bounds for armax models from records with bounded errors in variables. Int. J. Control 1993; 57(1):225-235.

33. Cerone V, Piga D, Regruto D. Improved parameters bounds for set-membership EIV problems. International Journal of Adaptive Control and Signal Processing 2011; 25(3):208-227.

34. Cerone V, Piga D, Regruto D. Set-membership EIV identification through LMI relaxation techniques. Proc. of the American Control Conference 2010, 2010; 2158-2163.

35. Cerone V, Piga D, Regruto D. Bounding the parameters of linear systems with stability constraints. Proc. of the American Control Conference 2010, 2010; 2152-2157. 